\section{Metabolite Findings in Tumefactive Demyelinating Lesions Utilizing Short Echo Time Proton Magnetic Resonance Spectroscopy}

The February 2007 issue of the American Journal of Neuroradiology reported the findings of A. Ciafoni et al. ${ }^{1}$ In this article, the authors reported the approach of examining glutamate/glutamine peaks in ${ }^{1} \mathrm{H}-\mathrm{MR}$ (proton) spectroscopy to increase the specificity in differentiating tumefactive demyelinating processes from neoplasms. The thought of using MR spectroscopy in this situation is attractive because the current clinical approach may warrant a lumbar puncture and even biopsy. Although we commend the authors' enthusiasm for this technique, the paper lacks statistical significance and contains much bias to support the authors' claim that MR spectroscopy "can be helpful in the noninvasive diagnosis of acute demyelinating diseases"1 when elevated glutamate/glutamine peaks are identified. We believe that this article should be represented as an idea backed by case reports and not be considered as original research.

First, the authors never fully give the inclusion or exclusion criteria for how the 4 patients in the study were chosen. Were any patients excluded from the study, and, if so, why? They state that the spectroscopy was performed on 4 patients with neurologic symptoms and a diagnosis of parenchymal mass lesions on MR imaging. It seems unlikely that the first 4 patients who entered the study had the diagnosis of tumefactive multiple sclerosis. There must have been some patients originally selected who ended up with the diagnosis of a neoplasm. Why were these spectroscopy results not included in the study? They fail to notify the reader if the diagnosis was already known at the time of selection and if the interpreter of the spectral data was blinded. Obviously, these effects can cause bias, which would alter the outcome of the study. The potential for bias is evident if one examines the 2.1 to 2.5 region of the spectra where complex, unresolved resonances produce a "shoulder" of variable slope downfield from the $\mathrm{N}$-acetylaspartate resonance. The fluctuations in the spectral intensity in this region are similar to the background noise (eg, Fig 2), which makes the assignment of a single peak height for the entire range challenging. Unfortunately, the authors have not described in detail their method for determining $\beta, \gamma$-Glx peak heights, and they have not documented the accuracy of the method for detecting changes in glutamine/glutamate levels (eg, in phantoms).

Second, no control is used. Referencing the article by Majos et $\mathrm{al}^{2}$ is not a substitute for using a control group with known neoplasms. Showing that a neoplasm does not increase the glutamate/glutamine peak would have added significantly to this article. Also, other demyelinating diseases are not included.

Finally, no statistical inference can be made in a study with only 4 patients; the study lacks statistical power.

For these reasons, we believe that this study should not be represented as original research, but as a novel idea with associated case reports to back up the need for more comprehensive research in this area. The facts in this study are not significant enough to suggest that MR spectroscopy "can be helpful" in distinguishing between tumefactive demyelinating diseases and neoplasm.

\section{References}

1. Cianfoni A, Niku S, Imbesi SG. Metabolite findings in tumefactive demyelinating lesions utilizing short echo time proton magnetic resonance spectroscopy. AJNR Am J Neuroradiol 2007;28:272-77

2. Majos C, Julia-Sape M, Alonso J, et al. Brain tumor classification by proton MR spectroscopy: comparison of diagnostic accuracy at short and long TE. AJNR Am J Neuroradiol 2004;25:1696-704

Marc Kalis

Department of Radiology Jackson Memorial Hospital

Miami, Fla

Brian C. Bowen

Robert M. Quencer

Department of Radiology

University of Miami School of Medicine

Miami, Fla

DOI 10.3174/ajnr.A0669

\section{Reply:}

Thank you for your comment and interest in our article. Our study was a retrospective evaluation of patients with tumefactive multiple sclerosis (MS), where the interesting finding of elevated glutamine/ glutamate (Glx) was noted on MR spectroscopy. As a retrospective study, only patients with the diagnosis of tumefactive MS were included, and the MR spectra were analyzed. Although this observation was not statistically significant given the limited number of patients in our study, we believe this finding of elevated Glx may be an appropriate differentiating metabolite in the interpretation of an MR spectroscopic examination of a mass lesion. Without doubt, a prospective study of all spectroscopic examinations performed at a given institution would supply more plausible evidence. Also, we fully agree that a major limitation of the article was the small number of patients; however, given the relatively rare incidence of this particular disease entity, statistically significant numbers of patients are difficult to obtain. ${ }^{1}$ In addition, given the retrospective nature of the study, a small control group of patients with neoplasms from our institution were not specifically included, but solid evidence regarding their typical spectra was provided by a very large published group of 151 patients with neoplasms. ${ }^{2}$

Our intention was not to suggest concrete evidence regarding this finding of elevated Glx in all possible cases and that it will be the sole differentiating factor between tumefactive MS and neoplasm, but to inform the reader of this interesting observation. Hopefully, as more cases are evaluated, a preponderance of additional evidence may be accumulated to further substantiate our finding. Furthermore, we specifically intended to notify the reader of the importance of obtaining short echo time (TE) spectra in addition to the usual acquisition of long TE spectra when a mass lesion is encountered. Although saving time during an MR examination is a noteworthy goal, the lack of obtaining metabolite information with short T1 and T2 on the long TE spectra alone results in a limited chemical evaluation of the pathologic entity and may suggest the wrong diagnosis.

As noted in the article, exact determination of the integration of the area under the Glx complex peak is prone to error, given the adjacent and usually incorporated $\mathrm{N}$-acetylaspartate (NAA) peak as well as the multiple spikes produced by the Glx metabolites. At present, the established method with a $1.5 \mathrm{~T}$ scanner is to choose the highest peak in the complex from 2.1 to $2.5 \mathrm{ppm}$ (which is usually the shoulder adjoining the NAA peak) and compare that peak height with the peak height of creatine. A ratio of 0.5 or greater is considered abnormal. ${ }^{3}$ With improved peak separation at $3 \mathrm{~T}$, more accurate quantitative methods to determine Glx should become standard practice.

We regret any misunderstanding that may have resulted but can only hope this publication prompts additional patients with tumefac- 